\title{
New catalogue of blue stragglers in open clusters ${ }^{\star}$
}

\author{
J. A. Ahumada and E. Lapasset
}

\author{
Observatorio Astronómico, Universidad Nacional de Córdoba, Laprida 854, 5000 Córdoba, Argentina \\ e-mail: [javier; lapasset]@oac.uncor.edu
}

Received 25 November 2005 / Accepted 26 September 2006

ABSTRACT

\begin{abstract}
We present a catalogue of blue-straggler candidates in galactic open clusters. It is based on the inspection of the colour-magnitude diagrams of the clusters, and it updates and supersedes the first version (Ahumada \& Lapasset 1995). A new bibliographical search was made for each cluster, and the resulting information is organised into two tables. Some methodological aspects have been revised, in particular those concerning the delimitation of the area in the diagrams where the stragglers are selected. A total of 1887 blue-straggler candidates have been found in 427 open clusters of all ages, doubling the original number. The catalogued stars are classified into two categories mainly according to membership information.
\end{abstract}

Key words. catalogs - open clusters and associations: general - stars: blue stragglers

\section{Introduction}

Blue stragglers are stars that seem to stay on the main sequence longer than is estimated by the standard theory of stellar evolution. They have been identified in star clusters, dwarf galaxies, and in the field. There is convincing evidence for the reality of the phenomenon, although more than one theory appears to be necessary to explain the observations. Their presence raises interesting issues for the evolution of stars and stellar systems.

In this paper we present a catalogue of blue-straggler candidates in galactic open clusters. It is based on the inspection of colour-magnitude diagrams and is an update of the work introduced by Ahumada \& Lapasset (1995), to which we refer for an early bibliography on the topic. Here at the start, we wish to draw attention to the reviews by Stryker (1993) and Bailyn (1995), as well as volume 53 of the A.S.P. (Saffer 1993).

Recent observational and theoretical results about blue stragglers are summarised in the rest of this section. The procedure for identifying the candidates is explained in Sect. 2. The description of the catalogue itself is found in Sect. 3. Some points of interest about the collected data are given in Sect. 4 , followed by the conclusions in Sect. 5 .

\subsection{Observations}

\subsubsection{Field blue stragglers}

These stars seem to belong to the thick disc or halo population according to their metallicity or kinematics, but they have relatively high mass. They can be identified by means of the relation between the $(B-V)$ index of the turnoff and the metallicity of the coeval, metal-poor, thick disc and halo populations (Carney et al. 1994): in this way, the authors find four new candidates. Several other candidates are singled out among highvelocity stars from their lithium abundances by Glaspey et al. (1994), and Andrievsky et al. (1995) spectroscopically analyse

^ The whole catalogue (Tables 8,9 , notes, and references) is only available in electronic form at the CDS via anonymous ftp to cdsarc.u-strasbg.fr $(130.79 .128 .5)$ or via http://cdsweb.u-strasbg.fr/cgi-bin/qcat? J/A+A/463/789 seven field stragglers. Two-thirds out of a group of sixty-two low-metallicity stars are binaries, of which about half may be blue stragglers (Preston \& Sneden 2000). These could have resulted from mass transfer events; in globular clusters, this kind of long-period binaries would not have survived. Mass transfer is also claimed for the stragglers HR 4657 (Fuhrmann \& Bernkopf 1999) and HD 115071 (Lloyd \& Stickland 2001). Ryan et al. (2001) study four lithium-deficient stars: this feature would identify stragglers produced by mass exchange in close binaries. Seven out of ten field straggler candidates show evidence of binarity (Carney et al. 2001); moreover, five of these are deficient in lithium, while two of the remaining stars have normal abundance. Spectroscopic analysis of metal-poor stars (Sneden et al. 2003) and of radial-velocity studies of metal-poor field blue stragglers (Carney et al. 2005) suggest that the observed abundances (including the lack of lithium) are consistent with stable mass transfer during the asymptotic-branch stage of the primaries, which are now unseen white-dwarf stars. It is worth pointing out that these low-lithium binaries show higher rotational velocities than do stars of the same temperature (Preston $\&$ Sneden 2000; Ryan et al. 2001; Carney et al. 2005). Regarding the link between the SX Phoenicis (SX Phe) variables and field blue stragglers, there is the work by Bernstein et al. (1995) on the discovery of one such variable in the halo, and those by Preston \& Landolt (1998, 1999), where several variables are also found to be binaries. Some metal-poor, main-sequence field-A stars are shown to exhibit thick-disc kinematics (Girard et al. 2004), which supports their interpretation of them as thick-disc blue stragglers.

\subsubsection{Open clusters}

Work has continued on the well-known blue-straggler populations of "classic" open clusters, as in the list that follows. NGC 2682 (M 67): assessment of the importance of the straggler population in the integrated UV spectrum (Landsman et al. 1998); discussion of the composition signatures of the cluster that could help to distinguish between mechanisms for the formation of stragglers (Shetrone \& Sandquist 2000); search for variability among the stragglers (Sandquist \& Shetrone 2003). 
$N G C$ 188: analysis of the degree of concentration of the stragglers (Dinescu et al. 1996). NGC 2632: spectroscopic analysis of stragglers (Andrievsky 1998; Burkhart \& Coupry 1998). NGC 6791: search for contact binaries that could be related to the stragglers (Ruciński et al. 1996). NGC 7789: spectroscopic study of its stragglers (Schönberner et al. 2001). Studies of other clusters include the identification of the straggler populations in Berkeley 20 (MacMinn et al. 1994) and Berkeley 32 (Richtler \& Sagar 2001); the search for variables among the stragglers in Berkeley 39 (Kałużny et al. 1993), Collinder 261 (Mazur et al. 1995), Berkeley 81, Berkeley 99, NGC 6603, and NGC 7044 (Sagar \& Griffiths 1998); spectroscopic analysis of stragglers in NGC 3496, NGC 6475, and NGC 6633 (Andrievsky et al. 2000), and NGC 3114 (González \& Lapasset 2001); identification of $\mathrm{X}$-sources among stragglers in IC 4651 (Belloni \& Tagliaferri 1998) and NGC 752 (Belloni \& Verbunt 1996); analysis of possible links between clump stars and stragglers in NGC 6819 (Rosvick \& VandenBerg 1998). There have also been studies of specific stragglers: the eclipsing binary D266 in NGC 2354 (Lapasset \& Ahumada 1996); the binary HR 3147 in NGC 2516 (Dachs \& Hummel 1996); the X-ray sources F124 (van den Berg et al. 2004) and F131 (Sandquist et al. 2003; van den Berg et al. 1999, 2001, 2004) in M 67; the short-period eclipsing precataclysmic binary V471 Tau in the Hyades (O’Brien et al. 2001).

\subsubsection{Globular clusters}

It is perhaps in this field of research where the advances in the knowledge of the blue stragglers have been most remarkable. A massive amount of new data, mainly photometric, is increasingly available, emphasising the importance of the populations of globular cluster stragglers. A new, homogeneous catalogue of about 3000 of these stars in 56 clusters has recently been presented by Piotto et al. (2004).

Among the studies of blue stragglers in globular clusters, we note the following: 47 Tucanae (Ferraro et al. 2001), IC 4499 (Ferraro et al. 1995), M 3 (Ferraro et al. 1997), M 13 (Cohen et al. 1997), M 53 (Rey et al. 1998), M 55 (Richter et al. 1999), NGC 5053 (Sarajedini \& Milone 1995), NGC 6229 (Borissova et al. 1999), NGC 6366 (Harris 1993), NGC 6553 (Zoccali et al. 2001), NGC 6752 (Sabbi et al. 2004), $\omega$ Centauri (Lyngå 1996), Palomar 13 (Siegel at al. 2001; Clark et al. 2004). They are present in very dense clusters like M30 (Guhathakurta et al. 1998), as well as in low-density ones such as NGC 6712 (Paltrinieri et al. 2001) or NGC 288 (Bellazzini et al. 2002). In all these clusters the number of blue stragglers is nothing short of remarkable, reaching an amazing count of more than 300 in M 80 (Ferraro et al. 1999). So large a population may be indicating that the cluster is on the verge of the core collapse.

Blue stragglers have also been identified in the populous young $\left(10^{6}-10^{7} \mathrm{yr}\right)$ clusters of the Magellanic Clouds, such as NGC 330 (Grebel et al. 1996, who argue that stragglers may be common among such clusters) in the SMC, or NGC 1805 and NGC 1818 in the LMC (Johnson et al. 2001); and in old $\left(>10^{10} \mathrm{yr}\right.$ ) extragalactic clusters, such as NGC 121 (Shara et al. 1998) in the SMC and Hodge 11, NGC 1466, and NGC 2257 in the LMC (Johnson et al. 1999).

Individual blue stragglers in globular clusters include: an eclipsing binary in $\omega$ Cen (Helt et al. 1993), a short-period eclipsing binary in M 5 (Yan \& Reid 1996), an SX Phe variable in NGC 6752 (Rubenstein 1997), six SX Phe variables in 47 Tuc (Gilliland et al. 1998; Bruntt et al. 2001), an eclipsing star in Terzan 5 (Edmonds et al. 2001), a W UMa star in NGC 3201 (von Braun \& Mateo 2002), an X-ray source straggler in M 22
(Webb et al. 2004). It is also worth mentioning the first determination of the mass of a blue straggler in a globular cluster, the star BSS 19 in 47 Tuc (Shara et al. 1997), as well as the first proof of circumstellar discs around blue stragglers in three globular clusters (De Marco et al. 2004).

\subsection{Theories}

These are the most frequently cited theories proposed to explain these stars:

1. They are post-main-sequence stars, perhaps horizontalbranch stars, that happen to appear above the turnoff. Although there could be some such cases (cf. D'Antona et al. 1995; Peterson \& Green 1998), most evidence points to the stragglers as main-sequence stars for a number of reasons: first, their surface gravities and effective temperatures (Strom \& Strom 1970; Eggen 1979); second, the similar concentration shown by the straggler and binary populations in several clusters (Mathieu \& Latham 1986); third, direct mass determinations, e.g., the star BSS 19 in 47 Tuc has a mass twice that of the turnoff of the cluster (Shara et al. 1997).

2. They are stars formed after the bulk of the system to which they belong (Eggen \& Iben 1988, 1989). Opposed to this hypothesis is the general absence of observational evidence of star formation indicators, such as dark nebulae or T Tauri stars, associated with the stragglers.

3. They are members of binary systems that have gained mass from their initially more massive companions, after these have reached an appropriate size in the course of their evolution (McCrea 1964). There is good observational evidence that supports this theory in some cases. For example, there are the binaries $\theta$ Carinae in the young open cluster IC 2602, F190 in the old open cluster M 67, and NJL 5 in the globular cluster $\omega \mathrm{Cen}$, as well as a number of X-ray sources in diverse systems, that are stragglers.

4. They are stars that have extended main-sequence lives due to some non-standard mechanism of internal mixing (Finzi \& Wolf 1968; Wheeler 1979). Rotation, magnetic fields, or a near companion would be able to induce internal currents that might carry fresh hydrogen to the core, thus modifying the normal evolution of the star. It has been argued that this mechanism can produce stragglers chiefly in young and intermediate-age clusters (Abt 1985) because many of them are peculiar A stars; these peculiarities would be manifestations of the internal mixing. This idea, which has also been applied to stragglers in OB associations (Mathys 1987), still has to explain why only a few stars are affected.

5. They are the products of collisional mergers of two stars (Hills \& Day 1976). This may be a suitable mechanism for stragglers in the core of dense globular clusters such as 47 Tuc (Guhathakurta et al. 1992).

6. They are contact binaries whose components have merged. Because some time is required for a detached binary to evolve into contact, these stragglers should only appear in old clusters. According to Mateo et al. (1990), the minimum time needed to make contact is between 1 and 5 Gyr if the initial periods are around 3 days. Stars such as FK Comae variables and anomalous cepheids could result from these mergers. It is worth pointing out, however, that the percentage of binaries with such short periods is small (Duquennoy \& Mayor 1991).

7. They are the product of collisional mergers between binaries, or between single stars and binaries (Leonard 1989). 
This mechanism is plausible in high-density environments, like many globular clusters, but the possibility cannot be discarded that a small fraction of the stragglers in low-density systems are formed in this way (Leonard \& Linnell 1992; Sandquist 2005).

Among the recent works that seek to confirm or reject these theories we can mention the following:

- Simulation of binary evolution in young open clusters (Pols \& Marinus 1994): the number and properties of the synthetic stragglers are consistent with the observations of clusters younger than $\sim 3 \times 10^{8} \mathrm{yr}$.

- Spectroscopy of stragglers in open clusters (Schönberner \& Napiwotzki 1994): internal mixing is not a suitable mechanism.

- Discussion of a scenario for the formation of some stragglers of the globular cluster M 15 (D'Antona et al. 1995): they may result from the merger of helium white dwarfs.

- Simulation of the formation of blue stragglers from direct collisions of main-sequence stars (Lombardi et al. 1995): the stragglers do not show any overabundance of helium.

- Discussion of the rotational rates of collisional blue stragglers (Leonard \& Livio 1995): a high rotation velocity would not be an identification mark of such stars.

- Study of the dispersion of binaries in globular clusters (Bacon et al. 1996): they dominate in low-density clusters, while in denser systems the star-binary collisions predominate.

- Modelling of collisional blue stragglers in globular clusters (Sills \& Bailyn 1999): the number and distribution of the stragglers in the colour-magnitude diagrams are determined by the current dynamical state and population of the clusters. More analyses of the formation of, and mixing in, collisional globular-cluster stragglers: Sills et al. (1995), Sandquist et al. (1997), Ouellette \& Pritchet (1998), Lombardi et al. (2002).

- Discussion of high-resolution spectra of stragglers in M67 (Shetrone \& Sandquist 2000): the abundance of CNO elements may be a better index for distinguishing collisional stragglers from those resulting from mass transfer in close binaries.

- Modelling of the blue straggler population of M67 (Hurley et al. 2001): the cluster environment and the evolution of the binaries must be taken into account when reproducing the observed straggler population.

- Simulation of the evolution of an open cluster (Portegies Zwart et al. 2001): two different straggler populations are formed.

- Discussion of a possible scenario for the formation of oscillating blue stragglers in globular clusters and dwarf galaxies (Santolamazza et al. 2001): the properties of the SX Phe variables can help to constrain the evolution of the stragglers.

- Theoretical study of the pulsating variables SX Phe that are, in turn, blue stragglers in open clusters (Templeton et al. 2002): the period-luminosity relation is not affected by a possible complete mixing of the stragglers if these are the result of collisions.

- Analysis of the frequency of primordial binaries and stragglers in the globular cluster NGC 288 (Bellazzini et al. 2002): the production of stragglers by the evolution of close binaries can be very efficient, even in low-density clusters.

- Simulation of mass transfer in stragglers, particularly star F 190 in M 67 (Chen \& Han 2004): these stars show abnormalities in the $\mathrm{CNO}$ abundances.
- Discussion of the possible presence of circumstellar discs around some stragglers (De Marco et al. 2004): a magnetic mechanism is required for losing the large angular momentum imparted by the initial collision event.

- Analysis of the spatial distribution of the blue straggler population of 47 Tuc (Mapelli et al. 2004): a complex scenario involving collision-generated as well as isolated, peripheral interacting binaries is required.

- Discussion of the observed populations of globular cluster stragglers (Piotto et al. 2004): there is a strong anticorrelation between the straggler frequency (relative to horizontal-branch stars) and the cluster-integrated magnitude (i.e., mass); there is also a weaker anticorrelation with the cluster central density. The first trend is confirmed for the lowest-density clusters by Sandquist (2005): stellar collisions still produce a significant fraction of the blue stragglers in low-luminosity star clusters, due to the long-term survival of wide binaries.

The observational evidence and the theoretical efforts thus point to a rather complex scenario, since it is apparent that more than one mechanism is needed to produce the blue stragglers, even in the same cluster. It is probable, however, that such mechanisms are among those already proposed, and it remains to be seen what their relative efficiency is in each kind of stellar system where the stragglers are observed.

\section{Search for blue stragglers in open clusters}

We look for blue-straggler candidates in the colour-magnitude diagrams of galactic open clusters. Our first catalogue of such stars (Ahumada \& Lapasset 1995, hereafter AL95) takes the bibliography on open clusters up to 1993 into account. As a great deal of new information has been added since then, it is befitting to carry out a new compilation of data. In addition, some methodological differences with AL95 call for a complete revision of the previous search, so this catalogue is essentially a new work, intended to supersede the first one.

\subsection{Colour-magnitude diagrams of open clusters}

\subsubsection{Source of the photometry}

In AL95 we commented that the available cluster diagrams were in general only those in print, which implied lack of homogeneity due to the different scales used by the authors in their graphics. There was also the impossibility of manipulating these diagrams whether to restrict the number of stars plotted or to superpose isochrones to simplify the analysis. To this we must add the intrinsic photometric uncertainties and the disposition of the authors to clean their diagrams of non-members, or to correct them by differential reddening.

Now, however, we have a useful tool: the Open Cluster Database WEBDA ${ }^{1}$ (Mermilliod \& Paunzen 2003). The database information on each cluster includes the tables of photometric data in electronic form, ready for plotting. This allows us to draw the colour-magnitude diagrams with the stars we wish to include, on the same scale for all clusters, and with the theoretical curves that we regard necessary. All the photometric studies of the cluster are available, permitting us a quick comparison among them, something not always possible before. Finally, there is additional information on each cluster star, which may cast light on its character as a blue straggler.

\footnotetext{
${ }^{1}$ http://www. univie.ac .at/webda
} 
Table 1. Open clusters with entries in AL95 but not included in the present catalogue.

\begin{tabular}{llll}
\hline \hline Basel 1 & Basel 4 & Basel 5 & Basel 7 \\
Basel 11a & Basel 11b & Basel 18 & Berkeley 94 \\
Bochum 4 & Bochum 5 & Bochum 11 & Bochum 12 \\
Bochum 13 & Bochum 15 & Collinder 173 & Collinder 197 \\
Collinder 240 & Collinder 285 & Collinder 347 & Collinder 469 \\
CV Mon & Czernik 29 & Haffner 15 & Haffner 20 \\
IC 1442 & Lynga 14 & Markarian 6 & NGC 189 \\
NGC 1605 & NGC 2254 & NGC 2286 & NGC 2395 \\
NGC 2423 & NGC 2579 & NGC 2925 & NGC 3330 \\
NGC 6208 & NGC 6546 & NGC 6683 & NGC 6802 \\
NGC 7031 & NGC 7062 & NGC 7226 & NGC 7245 \\
NGC 7762 & Pismis 17 & Ruprecht 18 & Ruprecht 92 \\
Ruprecht 93 & Ruprecht 97 & Ruprecht 108 & Sher 1 \\
Trumpler 22 & Trumpler 31 & Trumpler 33 & Trumpler 35 \\
Waterloo 6 & & & \\
\hline
\end{tabular}

\subsubsection{Selection of the photometry}

For a given cluster, there may be several available photometric studies. It is necessary, then, to make a selection with unified criteria.

1. Fundamentally, Johnson $U B V$ photometric studies were taken into account, especially the $V$ vs. $(B-V)$ diagrams. Colour-colour diagrams were analysed, if available, to help in membership considerations. As an alternative, $V$ vs. $(V-I)$ diagrams were considered when there were no $B V$ data. Clusters only observed in systems other than UBVI have been excluded; in particular, those clusters listed in AL95 that still have only $R G U$ observations do not have entries now.

2. Accuracy of the photometry. In general, bright clusters have good photoelectric photometry, which has been used. Otherwise, we prefer the more accurate CCD studies over the photographic ones. Nevertheless, since there are still very good photographic data, they are used if pertinent, as in the case of NGC 188. Colour-magnitude diagrams that, in our opinion, are photometrically poor have not been examined even if they belong to the sole study of a cluster.

3. Completeness of the photometry. Studies that cover larger areas have been preferred.

4. Colour-magnitude diagrams improved by dereddening or membership considerations have been preferred.

Stragglers were searched for again in all clusters for the sake of homogeneity. There are clusters, however, that have entries in AL95 but not here, fundamentally because we now judge their photometry as being very poor, and not improved in the intervening years. These clusters (57 in total) are listed in Table 1. On the other hand, the clusters that appear for the first time in the catalogue ( 85 in total) are in Table 2 . Note that the clusters listed in these two tables may or may not have stragglers.

\subsection{Blue stragglers in open clusters}

\subsubsection{Identification of the stragglers}

The tradional definition of a blue straggler can be summarised as follows: it is a star member of the cluster that in the colourmagnitude diagram appears blueward and above the turnoff, on or near the zero-age main sequence (ZAMS). In practice, however, there are issues to consider: (i) not all clusters have
Table 2. Open clusters that have entries for the first time in this catalogue.

\begin{tabular}{llll}
\hline \hline Berkeley 7 & Berkeley 12 & Berkeley 14 & Berkeley 17 \\
Berkeley 18 & Berkeley 20 & Berkeley 21 & Berkeley 22 \\
Berkeley 23 & Berkeley 28 & Berkeley 29 & Berkeley 30 \\
Berkeley 31 & Berkeley 33 & Berkeley 58 & Berkeley 60 \\
Berkeley 64 & Berkeley 66 & Berkeley 69 & Berkeley 70 \\
Berkeley 79 & Berkeley 81 & Berkeley 99 & Berkeley 104 \\
Bochum 2 & Bochum 6 & Bochum 7 & Collinder 69 \\
Collinder 74 & Collinder 110 & Collinder 261 & Feinstein 1 \\
Haffner 17 & Hogg 22 & King 5 & King 6 \\
King 7 & King 10 & King 15 & NGC 133 \\
NGC 366 & NGC 1220 & NGC 1348 & NGC 1798 \\
NGC 2126 & NGC 2141 & NGC 2192 & NGC 2259 \\
NGC 2304 & NGC 2311 & NGC 2467 & NGC 2627 \\
NGC 2658 & NGC 2849 & NGC 4815 & NGC 5999 \\
NGC 6005 & NGC 6253 & NGC 6268 & NGC 6404 \\
NGC 6514 & NGC 6583 & NGC 6631 & NGC 7044 \\
NGC 7419 & Pismis 2 & Pismis 3 & Pismis 7 \\
Pismis 16 & Pismis 18 & Ruprecht 46 & Ruprecht 55 \\
Ruprecht 67 & Ruprecht 75 & Ruprecht 115 & Ruprecht 119 \\
Ruprecht 120 & Ruprecht 130 & Stock 1 & Stock 7 \\
Tombaugh 2 & Trumpler 5 & Trumpler 11 & Trumpler 37 \\
vdB-Hagen 66 & & & \\
\hline
\end{tabular}

membership studies of all their observed stars; (ii) many colourmagnitude diagrams show strong contamination by field stars; (iii) there may be distortions of the observed sequences caused by differential reddening or by stellar phenomena associated with rapid rotation; and (iv) the stragglers may not be located exactly on the ZAMS, since many of these stars have clearly evolved from the main sequence: cf., e.g., Fig. 4 of Mermilliod (1982) or Fig. 1 of Bailyn (1995).

Following AL95, we have assumed a cluster star to be a bluestraggler candidate if it appears in a definite area of the cluster colour-magnitude diagram. Figure 1 is a schematic diagram where the area of interest appears shaded. Its approximate limits are the ZAMS to the left, the turnoff colour to the right, and down, the magnitude at which the observed sequence of the cluster separates from the ZAMS. In principle, we did not adopt an upper magnitude, although most blue stragglers are fainter than the 2.5 mag-limit above the cluster turnoff. This is consistent with the maximum magnitude for a cluster straggler as predicted by the mass-transfer theory (McCrea 1964), but there are exceptions (cf. Sect. 2.2.2).

With these aspects in mind, the procedure for each cluster is as follows.

1. The most suitable photometric work is selected among those available, as explained in Sect. 2.1.2.

2. The photometric data are plotted in a colour-magnitude diagram.

3. Starting with the cluster parameters listed by the WEBDA and then making small corrections, the approximate fitting of a theoretical isochrone is then performed on the cluster sequence. In this task we used the isochrones published by Girardi et al. (2000) (i) because they have a small step in the logarithm of the age (0.05), which allows for a finer fitting of the sequences, and (ii) for the sake of consistency with our observational work (Ahumada 2003). The ZAMS by Bertelli et al. (1994), shifted by the same amounts as the isochrone, is added to the graph.

4. The stragglers are singled out in the colour-magnitude diagram. 


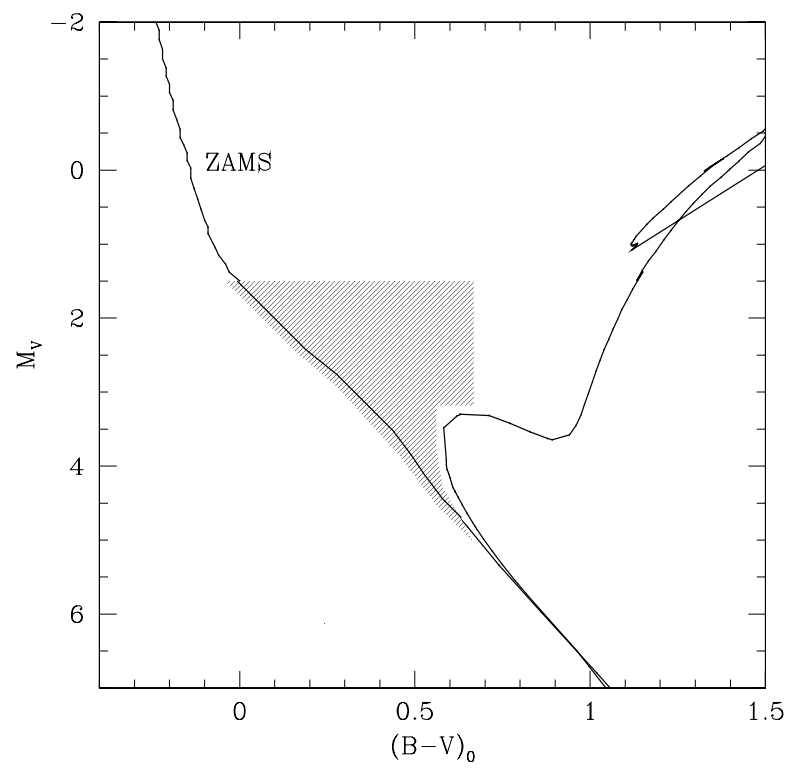

Fig. 1. Schematic colour-magnitude diagram for an old open cluster: the isochrone corresponds to $\log ($ age $)=9.8$. The blue straggler area is shaded.

In a more detailed level, several points must be taken into account.

1. The limits of the straggler area are only approximate: a star located slightly towards the blue (left) of the ZAMS can also be thought to be a straggler candidate, as well as stars brighter than $2.5 \mathrm{mag}$ above the turnoff, if there is no strong suspicion about their membership.

2. Any stars that are somewhat redder than the turnoff can also be considered: sometimes it is not easy to establish a clear red (right) limit, particularly in rich clusters, since their diagrams are very populated and often very contaminated. Occasionally we therefore imposed a slightly arbitrary cutoff in the colour $(B-V)$ or $(V-I)$ as the red limit of the blue straggler area. This limit is always given in the notes of the catalogue (Sect. 3.3).

3. The ability to draw isochrones sometimes proves very useful. Figure 1 schematically shows the evolutionary sequences of a very old cluster: it is evident that the separation between the ZAMS and the isochrone is wide enough to allow easy identification of the stragglers. For a young cluster, however, this is not so clear. Figure 2 shows another schematic colourmagnitude diagram, but now the isochrone corresponds to $\log ($ age $)=7.5$, with a long, nearly vertical section. In this case the blue straggler area is narrow; and without the help of the isochrones, it is easy to take as stragglers some stars that are bluer than the ZAMS, or bright stars that in fact are just on the isochrone. This is an important improvement over the methodology of AL95.

4. The choice of a set of isochrones implies the adoption of specific stellar models. For instance, the extension and shape of the "blue hook" depend on various factors, such as the amount of convective overshoot. Strictly speaking, the stars that, according to our isochrone fitting, appear on the blue hook, are not to be regarded as stragglers. One example is Fig. 3, which shows the $V$ vs. $(B-V)$ diagram of NGC 2632 (Praesepe). Note the group of stars that coincide with the blue hook; we do not regard them as straggler candidates, but as stars in the immediate post-main-sequence state, while the two catalogued blue stragglers are fully detached from the

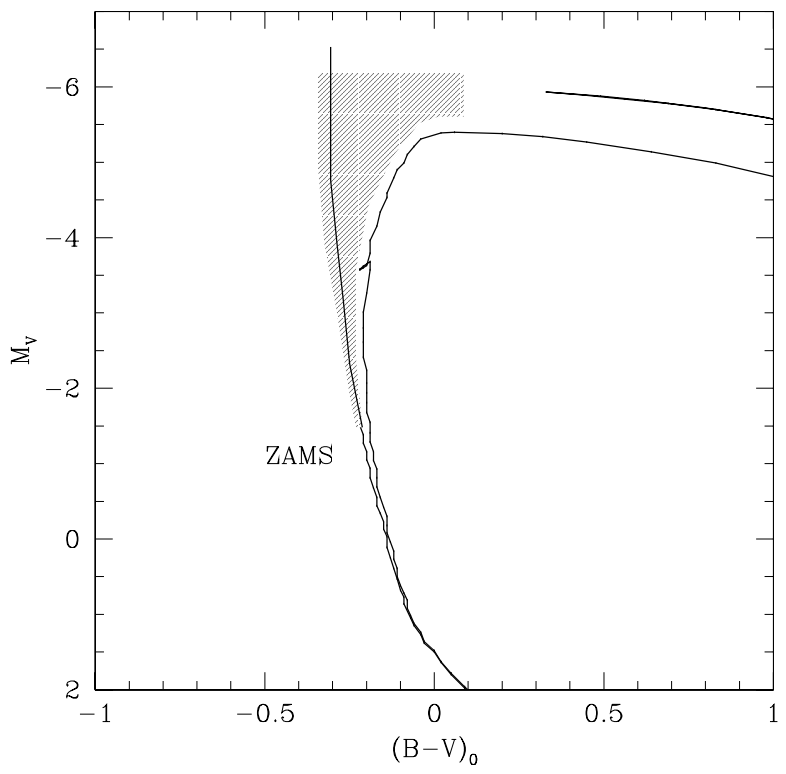

Fig. 2. Schematic colour-magnitude diagram for a young open cluster: the isochrone corresponds to $\log ($ age $)=7.5$. The blue straggler area is shaded.

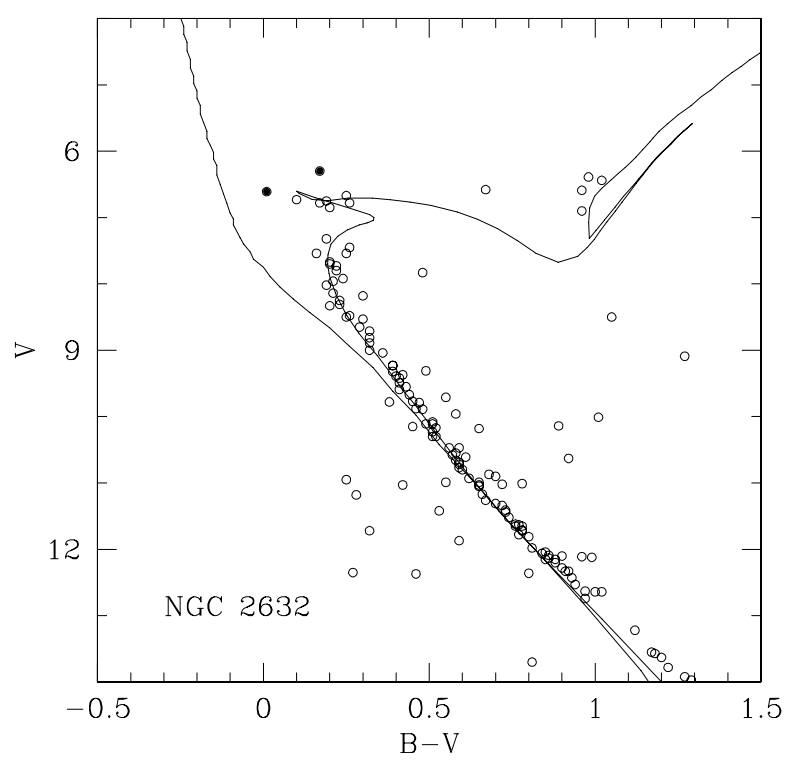

Fig. 3. Colour-magnitude diagram of NGC 2632. The photoelectric data of Johnson (1952) are plotted. The ZAMS from Bertelli et al. (1994) and the isochrone of solar metallicity and $\log ($ age $)=8.9$ by Girardi et al. (2000) are set at $E(B-V)=0.00$ and $(m-M)_{0}=6.25$. The two catalogued blue stragglers (solid circles) and the blue hook are seen clearly.

curves. Thus, in the selection of the stragglers, the models used in the fittings are involved. This is another difference to the procedure in AL95, where we only had an appreciation of the turnoff but generally not of the finer evolutionary sequences.

5. After a first selection of candidates, additional information on probability is taken from the database. If there is indication of a low $(<10 \%)$ membership probability, the star is deleted from the list. If an author has already established in his work that a star is a non-member, it is also deleted.

6. In very rich (and mostly old) clusters with a high number of straggler candidates, we restricted the selection up to a 


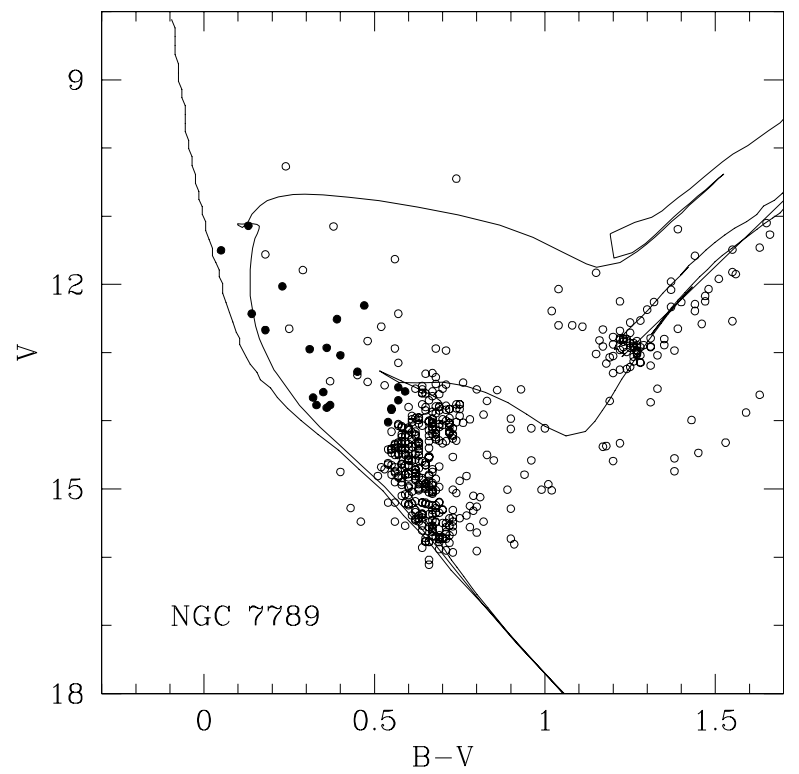

Fig. 4. Colour-magnitude diagram of NGC 7789. The photographic data of Burbidge \& Sandage (1958) are plotted. The solid circles represent the stragglers listed in our catalogue. From left to right, we show the solar-metallicity ZAMS of Bertelli et al. (1994) and the isochrones of Girardi et al. (2000) for $\log$ (age) $=8.45$ and 9.15; the curves are set at $E(B-V)=0.20$ and $(m-M)_{0}=11.42$. The massive stragglers are the two brightest.

certain radius around the cluster centre. Usually, we took this limit to be greater than the cluster apparent radius.

\subsubsection{Massive stragglers}

We can take advantage of using isochrones to identify those straggler candidates that exceed the limit given by the masstransfer theory (McCrea 1964). It is pertinent to remember that the 2.5-magnitude limit results from calculating the brightness of a star with a mass that doubles the brightness of a turnoff star; i.e., it is a hypothetical star that is the product of a complete merger of two turnoff stars. It is worth noting that this is not a photometric effect, since a simple calculation shows that the magnitude of an unresolved binary star with identical components is only $0.75 \mathrm{mag}$ above the magnitude of each component. This binary may appear as a straggler, but its components may not be such (cf., e.g., the case of V374 Car in NGC 2516, González \& Lapasset 2003). To find the massive stragglers, we started with the diagrams and isochrones used in Sect. 2.2.1. We plotted another isochrone whose turnoff is 2.5 mag brighter than the turnoff of the first isochrone; both curves are of course shifted by the same amounts in colour and magnitude. The stars located above the turnoff of - and outside the area delimited by - the younger isochrone are those regarded as potential massive stragglers. As an example, Fig. 4 shows the $V$ vs. $(B-V)$ diagram of NGC 7789 ; the isochrone that fits the main sequence is the one for $\log ($ age $)=9.15$ (with a turnoff magnitude $\left.V_{\mathrm{TO}} \simeq 14.4\right)$, and the second isochrone corresponds to $\log ($ age $)=8.45\left(V_{\mathrm{TO}} \simeq 11.9\right)$. The two known (Breger \& Wheeler 1980) massive stragglers are clearly seen above and to the left. The stars identified in this way are flagged with an $M$ in the second table of the catalogue (Sect. 3.2).
Table 3. Rich open clusters with many stragglers.

\begin{tabular}{llll}
\hline \hline Berkeley 12 & Berkeley 14 & Berkeley 17 & Berkeley 18 \\
Berkeley 21 & Berkeley 22 & Berkeley 31 & Berkeley 39 \\
Berkeley 66 & Berkeley 70 & Berkeley 99 & Collinder 110 \\
Collinder 261 & King 2 & King 11 & NGC 1193 \\
NGC 2141 & NGC 2158 & NGC 2194 & NGC 6253 \\
Pismis 2 & Pismis 3 & Ruprecht 46 & Trumpler 5 \\
\hline
\end{tabular}

\subsubsection{Classification of the blue stragglers}

Given the disparity in the information available for each straggler candidate, it is clear that all of them cannot have the same quality. In AL95 the stragglers were separated into three categories. Practice, however, has shown that this distinction is excessive, as it suffices to distinguish between bona fide blue stragglers and the rest. Those stars belonging to the first category have additional studies that suggest good membership probabilities. The others also appear in the straggler area defined in Sect. 2.2.1, but lack membership information. There is an exception to this general criterion, however, the stragglers at the centre of rich clusters. It has been shown, as in M 67 (Mathieu \& Latham 1986), that the stragglers often appear centrally concentrated. Thus we have decided to also put into the first category those stars that, when even lacking membership studies, satisfy the following: (i) they appear in clusters with a number of stars in the upper main sequence ( $N$, see Sect. 3.1) greater than 100 ; (ii) they are more than 10; (iii) they are located, with respect to the cluster centre, at up to a relative radius of 0.3 ; (iv) they are clearly differentiated from the cluster sequences; and (v) they are not too bright. The clusters whose stragglers were treated in this way are listed in Table 3.

As pointed out in AL95, some subjectivity comes into play when identifying and classifying the blue-straggler candidates. However, we believe that, on this occasion, we have introduced some elements that help to constrain the problem better and reduce some uncertainties in the procedure.

\section{Description of the catalogue}

The structure of the catalogue is the same as that of AL95, i.e., there is one table for open cluster data, a second table for blue straggler data, a file with additional notes, and another with the references. While the cluster parameters listed in AL95 were taken from Lyngå's Catalogue of Open Cluster Data (1987), in this case they are mainly those of the WEBDA. In doing so, we assume them to be the best parameters available. They are the equatorial coordinates (2000.0), the reddening $E(B-V)$, and the logarithm of the age. Note that this catalogue does not list the dereddened colour of the turnoff, as in AL95.

\subsection{First table: open cluster data (Table 8 available electronically)}

This table lists information on each open cluster examined, even those without stragglers; it contains ten columns:

(i) name of the cluster, listed by right ascension;

(ii) right ascension;

(iii) declination;

(iv) reddening;

(v) logarithm of the age; 
(vi) the cluster's apparent diameter. These have been taken mainly from Lyngå's (1987) catalogue, and are given in arcmin;

(vii) the number $N$, introduced in AL95, is the number of stars in the main sequence from the turnoff down two magnitudes. The number $N$ can be interpreted as a measure of the cluster richness. This counting was done in the same colour-magnitude diagram where the stragglers were identified;

(viii) the number $N_{\mathrm{BS}}$ is the total of straggler candidates found;

(ix) the reference of the photometry examined;

(x) an asterisk (*) refers to the notes (Sect. 3.3) for additional information.

\subsection{Second table: blue stragglers in open clusters (Table 9 available electronically)}

This table lists information on every straggler. It contains thirteen columns:

(i) name of the cluster, listed by right ascension;

(ii) identification of the straggler, as given by the photometric study from which the star was selected, i.e., the source of the listed magnitudes and colours; the stragglers are arranged according to these numbers;

(iii) identification of the straggler $(W E B D A)$. This is the common number given for each star in the WEBDA, to facilitate the cross-correlation of all the available information;

(iv) the straggler classification 1 or 2 (Sect. 2.2.3);

(v) the calification as a massive straggler, if pertinent, with an $M$ (Sect. 2.2.2);

(vi) $\quad V$ magnitude. Only observed (i.e., non dereddened) magnitudes and colours are listed;

(vii) $(B-V)$ index;

(viii) $(U-B)$ index.

(ix) $(V-I)$ index;

(x) type of photometry; this is described as: "pe" (photoelectric), "pg" (photographic), and CCD;

(xi) relative radius $r / R$, which gives the position of the straggler in the cluster. The distance $r$ from the cluster centre is calculated through the coordinates $(x, y)$ listed for each star in the WEBDA. The cluster centres are defined in the database. The radius $R$ is half the apparent diameter listed in the first table, column (vi);

(xii) reference of the photometry examined;

(xiii) an asterisk (*) refers to the notes for additional information.

\subsection{Notes (available electronically)}

This file gathers information that clarifies, complements, or simply adds to what is in the first two tables for the asterisked clusters, which are listed alphabetically. In particular, membership probabilities and spectra of the stragglers can be found here.

\subsection{References (available electronically)}

This is an autonomous reference system for the previous files. The references are arranged alphabetically, but in the tables and notes they are numbered.

\section{Some remarks on the collected data}

A total of 1887 blue-straggler candidates were identified in 427 galactic open clusters. As in AL95, the stragglers appear

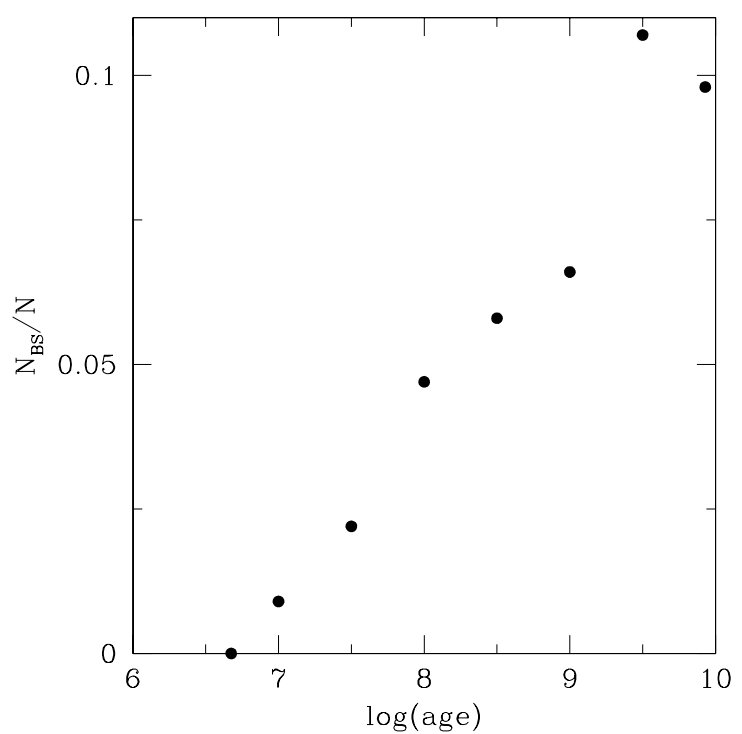

Fig. 5. Average number of blue stragglers, per main-sequence star and cluster.

in clusters of all ages. The number of clusters with at least one blue straggler of any category is 199 (46.6\% of the total). In AL95 this percentage was $57.7 \%$; the smaller number probably reflects more rigour in the selection of the candidates. A total of 200 stragglers was classified as $1(10.6 \%)$, and 1687 were classified as 2; in AL95 the candidates labelled 1 totalled 16.8\%. As said before, these figures must reflect more care in the process of classification; nevertheless, the net number of stragglers in the first class has indeed increased, because in AL95 it was 161.

That the absolute number of stragglers grows with the cluster age (cf., e.g., Tables 6 and 7, below) is expected, given the increasing masses of the oldest of the surviving clusters. But the trend of the relative number of stragglers $N_{\mathrm{BS}} / N$ vs. cluster age, shown in Fig. 5, is also always positive. An obvious explanation for Fig. 5 is the generation of blue stragglers from a physical mechanism whose productivity grows with time such as mass transfer in close binaries. There is a difference between Fig. 5 and its analogue in AL95 (Fig. 4); the old one showed two trends: one is approximately constant for young clusters and the second one growing with age. A probable explanation for the difference between the two figures is the selection of spurious candidates in young clusters that have not been included now, due to the better delimitation of the straggler area.

Figure 5 is insufficient, however, for further clarifying the phenomenon. In this sense, the number of stragglers found to show interesting features, such as binarity, variability, or spectral peculiarities, has clearly increased since AL95. Table 4 lists these stars, and notice the number of stragglers with special spectral features.

It is clear that the search for more peculiarities among the stragglers will be rewarding. The case of M67 is illustrative. Among the stragglers of this old (log $($ age $)=9.4)$ cluster, there are at least a W UMa binary (star 1036, Sandquist \& Shetrone 2003), two $\delta$ Sct variables (stars 1280 and 1284, Gilliland \& Brown 1992), an X-source and triple star (1082, van den Berg et al. 1999; Sandquist et al. 2003), another X-source (star 997, van den Berg et al. 2004), and several binaries (star 1284, Milone 1992; star 1434, Peterson et al. 1984; star 975, Landsman et al. 1998). While several of these stars can be explained by processes in close binaries, there are stragglers, such as star 977 (Mathys 1991), that may be the products of collisional mergers. All of this, and in only one cluster. 
Table 4. Peculiar blue stragglers (for the references, see the notes of the catalogue).

\begin{tabular}{|c|c|}
\hline Cluster & "Stragglers \\
\hline Berkeley 39 & Four eclipsing binaries and a $\delta$ Sct variable. \\
\hline Collinder 261 & Ten eclipsing binaries. \\
\hline Hyades & An Am star. \\
\hline IC 2602 & $\begin{array}{l}\text { The spectroscopic binary } \theta \text { Car has a period } 1.77 \\
\text { days and a spectrum Bp. }\end{array}$ \\
\hline IC 4651 & $\begin{array}{l}\text { Two probable binaries, one of them is an X-ray } \\
\text { source. }\end{array}$ \\
\hline IC 4725 & A Be straggler. \\
\hline IC 4756 & An Ap straggler. \\
\hline Melotte 111 & An $\alpha \mathrm{CVn}$ variable, of spectrum Ap. \\
\hline NGC 188 & A W UMa binary. \\
\hline NGC 752 & An X-ray source. \\
\hline NGC 884 & An Op straggler. \\
\hline NGC 1342 & An Ap straggler. \\
\hline NGC 2251 & An Ap straggler. \\
\hline NGC 2281 & An Ap straggler. \\
\hline NGC 2287 & A Bp straggler. \\
\hline NGC 2354 & An Algol, eclipsing binary. \\
\hline NGC 2422 & A Be straggler. \\
\hline NGC 2516 & V374 Car is a binary of spectrum $\mathrm{Be}$. \\
\hline NGC 2546 & An Ap straggler. \\
\hline NGC 2632 & An Ap straggler; another Am and binary. \\
\hline NGC 2682 & Several binaries, $\mathrm{X}$-sources, and $\delta$ Sct stars. \\
\hline NGC 3532 & A Bp straggler. \\
\hline NGC 4755 & А $\beta$ Cep variable. \\
\hline NGC 6025 & A Be straggler. \\
\hline NGC 6231 & An Op straggler. \\
\hline NGC 6425 & A Be straggler. \\
\hline NGC 6475 & A spectroscopic binary. \\
\hline NGC 6633 & A Bp straggler. \\
\hline NGC 6705 & A Bp straggler. \\
\hline NGC 6791 & An EW variable. \\
\hline NGC 6871 & A WR binary. \\
\hline NGC 7243 & An Ap straggler. \\
\hline NGC 7789 & An Ap star, and a $\delta$ Sct variable. \\
\hline
\end{tabular}

Table 5. Clusters with massive blue stragglers $\left(N_{\mathrm{M}}>5\right)$.

\begin{tabular}{lcccc}
\hline \hline Cluster & log (age) & $N_{\mathrm{BS}}$ & $N_{\mathrm{M}}$ & $N_{\mathrm{M}} / N_{\mathrm{BS}}$ \\
\hline Berkeley 14 & 9.2 & 94 & 9 & 0.10 \\
Berkeley 18 & 9.6 & 126 & 28 & 0.22 \\
Berkeley 21 & 9.3 & 51 & 10 & 0.20 \\
Berkeley 66 & 9.7 & 70 & 9 & 0.13 \\
Berkeley 70 & 9.7 & 64 & 12 & 0.19 \\
Melotte 66 & 9.5 & 35 & 8 & 0.23 \\
NGC 1798 & 9.2 & 24 & 6 & 0.25 \\
Ruprecht 46 & 9.6 & 62 & 16 & 0.26 \\
Trumpler 5 & 9.6 & 70 & 15 & 0.21 \\
\hline
\end{tabular}

On the other side, it can also be worth investigating the massive stragglers (Sect. 2.2.2), since they cannot be explained by the mass-transfer theory. We identified 148 stars in 26, mostly old (age $\geq 10^{9} \mathrm{yr}$ ), clusters. Table 5 lists the clusters with a number of massive straggler candidates $\left(N_{\mathrm{M}}\right)$ above 5 ; the relation $N_{\mathrm{M}} / N_{\mathrm{BS}}$ is also given; the mean value of this ratio is $\sim 0.2$. There are three young clusters whose sole straggler seems to be massive: NGC 1664, NGC 2353, and NGC 6494. These objects deserve to be examined as well.

Finally, it may be of interest to list those clusters with the largest absolute $\left(N_{\mathrm{BS}} \geq 20\right.$, Table 6$)$ and relative $\left(N_{\mathrm{BS}} / N \geq 0.1\right.$, Table 7) straggler populations. These clusters, all of them old
Table 6. Clusters with the largest absolute populations of blue stragglers $\left(N_{\mathrm{BS}} \geq 20\right)$.

\begin{tabular}{lcc|lcc}
\hline \hline Cluster & log (age $)$ & $N_{\text {BS }}$ & Cluster & $\log ($ age $)$ & $N_{\text {BS }}$ \\
\hline Berkeley 18 & 9.6 & 126 & Berkeley 17 & 10.1 & 31 \\
Berkeley 14 & 9.2 & 94 & King 2 & 9.8 & 30 \\
NGC 6791 & 9.6 & 75 & Pismis 3 & 9.0 & 30 \\
Berkeley 66 & 9.7 & 70 & NGC 6819 & 9.2 & 29 \\
Trumpler 5 & 9.6 & 70 & Collinder 110 & 9.2 & 27 \\
Berkeley 70 & 9.7 & 64 & King 11 & 9.2 & 27 \\
Ruprecht 46 & 9.6 & 62 & NGC 6253 & 9.7 & 27 \\
Collinder 261 & 10.0 & 54 & NGC 188 & 9.6 & 24 \\
Berkeley 21 & 9.3 & 51 & NGC 1798 & 9.2 & 24 \\
Pismis 2 & 9.1 & 46 & NGC 2141 & 9.2 & 24 \\
Berkeley 39 & 9.9 & 43 & NGC 2682 & 9.4 & 30 \\
NGC 2158 & 9.0 & 40 & NGC 7789 & 9.2 & 22 \\
Berkeley 32 & 9.5 & 37 & Berkeley 22 & 9.0 & 21 \\
NGC 7142 & 9.3 & 37 & Berkeley 31 & 9.3 & 20 \\
Melotte 66 & 9.5 & 35 & & & \\
\hline
\end{tabular}

Table 7. Clusters with the largest relative populations of blue stragglers $\left(N_{\mathrm{BS}} / N \geq 0.1\right)$.

\begin{tabular}{lcc|lcc}
\hline \hline Cluster & $\log ($ age $)$ & $N_{\mathrm{BS}} / N$ & Cluster & $\log ($ age $)$ & $N_{\mathrm{BS}} / N$ \\
\hline Ruprecht 46 & 9.6 & 0.26 & King 11 & 9.1 & 0.13 \\
Berkeley 70 & 9.7 & 0.22 & NGC 188 & 9.6 & 0.13 \\
NGC 7142 & 9.3 & 0.21 & Pismis 3 & 9.0 & 0.13 \\
Berkeley 66 & 9.7 & 0.19 & Berkeley 21 & 9.3 & 0.12 \\
Berkeley 14 & 9.2 & 0.17 & King 2 & 9.8 & 0.12 \\
NGC 2682 & 9.4 & 0.17 & NGC 2112 & 9.3 & 0.12 \\
NGC 7789 & 9.2 & 0.17 & NGC 2627 & 9.3 & 0.11 \\
Berkeley 17 & 10.1 & 0.16 & Pismis 2 & 9.1 & 0.10 \\
Berkeley 32 & 9.5 & 0.16 & Berkeley 81 & 9.0 & 0.10 \\
NGC 6005 & 9.1 & 0.16 & & & \\
\hline
\end{tabular}

(age $\geq 10^{9} \mathrm{yr}$ ), may turn out to be valuable objects for trying to model cluster and straggler populations and their evolution.

\section{Conclusions}

The present new catalogue of blue stragglers in open clusters arises from the necessity of updating our first version (AL95) and from two important facts: (i) some deficiencies or lack of precision detected in that catalogue and (ii) the great amount of new studies published since 1995, mainly related to the photometry of open clusters, in part summarised in Sect. 1.1.2. Concerning point (i), we tried to define more accurately what stars in the colour-magnitude diagram are to be regarded as bluestraggler candidates. Some subjectivity remains in this selection, but this is unavoidable. On the other hand, and as a question of simplicity, the blue stragglers have been classified this time into just two categories: those bona fide blue stragglers members of the clusters, and those whose membership condition must be confirmed. The fact that only a small percentage of stars belong to the first category indicates that much more observational work is required to define membership. Many open clusters also need better photometric data since some of them only possess old and imprecise observations. In summary, we have found 1887 blue straggler candidates, which doubles the number of our first catalogue.

It is our hope that this new version of our catalogue of blue stragglers will provide a suitable database for future investigations of these objects. There still remain many clusters with poor photometric data that deserve new studies with modern techniques. Also, as already pointed out in AL95, systematic 
spectroscopic radial velocities and photometric variability studies are needed to clarify the blue straggler phenomenon.

Acknowledgements. It is a pleasure to thank the referee, Dr. B. W. Carney, for his useful comments and suggestions. This research made use of the WEBDA database, operated at the Institute for Astronomy of the University of Vienna.

\section{References}

Abt, H. A. 1985, ApJ, 294, L103

Ahumada, J. A. 2003, Rev. Mex. Astron. Astrofis., 39, 41

Ahumada, J., \& Lapasset, E. 1995, A\&AS, 109, 375 (AL95)

Andrievsky, S. M. 1998, A\&A, 334, 139

Andrievsky, S. M., Chernyshova, I. V., \& Ivashchenko, O. V. 1995, A\&A, 297, 356

Andrievsky, S. M., Schönberner, D., \& Drilling, J. S. 2000, A\&A, 356, 517

Bacon, D., Sigurdsson, S., \& Davies, M. B. 1996, MNRAS, 281, 830

Bailyn, C. D. 1995, ARA\&A, 33, 133

Bellazzini, M., Fusi Pecci, F., Messineo, M., Monaco, L., \& Rood, R. T. 2002, AJ, 123, 1509

Belloni, T., \& Tagliaferri, G. 1998, A\&A, 335, 517

Belloni, T., \& Verbunt, F. 1996, A\&A, 305, 806

Bernstein, G. M., Knezek, P., \& Offutt, W. 1995, PASP, 107, 521

Bertelli, G., Bressan, A., Chiosi, C., Fagotto, F., \& Nasi, E. 1994, A\&AS, 106, 275

Borissova, J., Catelan, M., Ferraro, F. R., et al. 1999, A\&A, 343, 813

Breger, M., \& Wheeler, J. C. 1980, PASP, 92, 514

Bruntt, H., Frandsen, S., Gilliland, R. L., et al. 2001, A\&A, 371, 614

Burbidge, E. M., \& Sandage, A. 1958, ApJ, 128, 174

Burkhart, C., \& Coupry, M. F. 1998, A\&A, 338, 1073

Carney, B. W., Latham, D. W., Laird, J. B., \& Aguilar, L. A. 1994, AJ, 107, 2240

Carney, B. W., Latham, D. W., Laird, J. B., Grant, C. E., \& Morse, J. A. 2001, AJ, 122, 3419

Carney, B. W., Latham, D. W., \& Laird, J. B. 2005, AJ, 129, 466

Chen, X., \& Han, Z. 2004, MNRAS, 355, 1182

Clark, L. L., Sandquist, E. L., \& Bolte, M. 2004, AJ, 128, 3019

Cohen, R. L., Guhathakurta, P., Yanny, B., Schneider, D. P., \& Bahcall, J. N. 1997, AJ, 113, 669

Dachs, J., \& Hummel, W. 1996, A\&A, 312, 818

D’Antona, F., Vietri, M., \& Pesce, E. 1995, MNRAS, 272, 730

De Marco, O., Lanz, T., Ouellette, J. A., Zurek, D., \& Shara, M. M. 2004, ApJ, 606, L151

Dinescu, D. I., Girard, T. M., van Altena, W. F., Yang, T.-G., \& Lee, Y.-W. 1996, AJ, 111, 1205

Duquennoy, A., \& Mayor, M. 1991, A\&A, 248, 485

Edmonds, P. D., Grindlay, J. E., Cohn, H., \& Lugger, P. 2001, ApJ, 547, 829

Eggen, O. J. 1979, ApJS, 41, 413

Eggen, O. J., \& Iben, I. 1988, AJ, 96, 635

Eggen, O. J., \& Iben, I. 1989, AJ, 97, 431

Ferraro, F. R., Paltrinieri, B., Fusi Pecci, F., et al. 1997, A\&A, 324, 915

Ferraro, F. R., Paltrinieri, B., Rood, R. T., \& Dorman, B. 1999, ApJ, 522, 983

Ferraro, F. R., D’Amico, N., Possenti, A., Mignani, R. P., \& Paltrinieri, B. 2001, ApJ, 561, 337

Ferraro, I., Ferraro, F. R., Fusi Pecci, F., Corsi, C. E., \& Buonanno, R. 1995, MNRAS, 275, 1057

Finzi, A., \& Wolf, R. A. 1968, ApJ, 153, 865

Fuhrmann, K., \& Bernkopf, J. 1999, A\&A, 347, 897

Gilliland, R. L., \& Brown, T. M. 1992, AJ, 103, 1945

Gilliland, R. L., Bono, G., Edmonds, P. D., et al. 1998, ApJ, 507, 818

Girard, T. M., Dinescu, D. I., van Altena, W. F., et al. 2004, AJ, 127, 3060

Girardi, L., Bressan, A., Bertelli, G., \& Chiosi, C. 2000, A\&AS, 141, 371

Glaspey, J. W., Pritchet, C. J., \& Stetson, P. B. 1994, AJ, 108, 271

González, J. F., \& Lapasset, E. 2001, AJ, 121, 2657

González, J. F., \& Lapasset, E. 2003, A\&A, 404, 365

Grebel, E. K., Roberts, W. J., \& Brandner, W. 1996, A\&A, 311, 470

Guhathakurta, P., Yanny, B., Schneider, D. P., \& Bahcall, J. N. 1992, AJ, 104, 1790

Guhathakurta, P., Webster, Z. T., Yanny, B., Schneider, D. P., \& Bahcall, J. N. 1998, AJ, 116, 1757

Harris, H. C. 1993, AJ, 106, 604

Helt, B. E., Jorgensen, H. E., King, S., \& Larsen, A. 1993, A\&A, 270, 297

Hills, J. G., \& Day, C. A. 1976, ApL, 17, 87

Hurley, J. R., Tout, C. A., Aarseth, S. J., \& Pols, O. R. 2001, MNRAS, 323, 630

Johnson, H. L. 1952, ApJ, 116, 640

Johnson, J. A., Bolte, M., Stetson, P. B., Hesser, J. E., \& Somerville, R. S. 1999, ApJ, 527, 199
Johnson, R. A., Beaulieu, S. F., Gilmore, G. F., et al. 2001, MNRAS, 324, 367

Kałużny, J., Mazur, B., \& Krzemiński, W. 1993, MNRAS, 262, 49

Landsman, W., Bohlin, R. C., Neff, S. G., et al. 1998, AJ, 116, 789

Lapasset, E., \& Ahumada, J. 1996, A\&A, 314, 448

Leonard, P. J. T. 1989, AJ, 98, 217

Leonard, P. J. T., \& Linnell, A. 1992, AJ, 103, 1928

Leonard, P. J. T., \& Livio, M. 1995, ApJ, 447, L121

Lloyd, C., \& Stickland, D. J. 2001, A\&A, 370, 1026

Lombardi, J. C., Rasio, F. A., \& Shapiro, S. L. 1995, ApJ, 445, L117

Lombardi, J. C., Warren, J. S., Rasio, F. A., Sills, A., \& Warren, A. A. 2002, ApJ, 568,939

Lyngå, G. 1987, Catalogue of Open Cluster Data, Centre des Données Stellaires, Strasbourg

Lyngå, G. 1996, A\&AS, 115, 297

MacMinn, D., Phelps, R. L., Janes, K. A., \& Friel, E. D. 1994, AJ, 107, 1806

Mapelli, M., Sigurdsson, S., Colpi, M., et al. 2004, ApJ, 605, L29

Mateo, M., Harris, H., Nemec, J., \& Olszewski, E. 1990, AJ, 100, 469

Mathieu, R. D., \& Latham, D. W. 1986, AJ, 92, 1364

Mathys, G. 1987, A\&AS, 71, 201

Mathys, G. 1991, A\&A, 245, 467

Mazur, B., Krzemiński, W., \& Kałużny, J. 1995, MNRAS, 273, 59

McCrea, W. 1964, MNRAS, 128, 147

Mermilliod, J.-C. 1982, A\&A, 109, 37

Mermilliod, J.-C., \& Paunzen, E. 2003, A\&A, 410, 511

Milone, A. A. E. 1992, PASP, 104, 1268

O’Brien, M. S., Bond, H. E., \& Sion, E. M. 2001, ApJ, 563, 971

Ouellette, J. A., \& Pritchet, C. J. 1998, AJ, 115, 2539

Paltrinieri, B., Ferraro, F. R., Paresce, F., \& De Marchi, G. 2001, AJ, 121, 3114

Peterson, R. C., \& Green, E. M. 1998, ApJ, 502, L39

Peterson, R. C., Carney, B. W., \& Latham, D. W. 1984, ApJ, 279, 237

Piotto, G., De Angeli, F., King, I. R., et al. 2004, ApJ, 604, L109

Pols, O., \& Marinus, M. 1994, A\&A, 288, 475

Portegies Zwart, S. F., McMillan, S. L. W., Hut, P., \& Makino, J. 2001, MNRAS, 321,199

Preston, G. W., \& Landolt, A. U. 1998, AJ, 115, 2515

Preston, G. W., \& Landolt, A. U. 1999, AJ, 118, 3006

Preston, G. W., \& Sneden, C. 2000, AJ, 120, 1014

Rey, S.-C., Lee, Y.-W., Byun, Y.-I., \& Chun, M.-S. 1998, AJ, 116, 1775

Richter, P., Hilker, M., \& Richtler, T. 1999, A\&A, 350, 476

Richtler, T., \& Sagar, R. 2001, BASI, 29, 53

Rosvick, J. M., \& VandenBerg, D. A. 1998, AJ, 115, 1516

Rubenstein, E. P. 1997, PASP, 109, 933

Ruciński, S. M., Kałużny, J., \& Hilditch, R. W. 1996, MNRAS, 282, 705

Ryan, S. G., Beers, T. C., Kajino, T., \& Rosolankova, K. 2001, ApJ, 547, 231

Sabbi, E., Ferraro, F. R., Sills, A., \& Rood, R. T. 2004, ApJ, 617, 1296

Saffer, R. A. (ed.) 1993, Blue Stragglers, ASP Conf. Ser., 53

Sagar, R., \& Griffiths, W. K. 1998, MNRAS, 299, 1

Sandquist, E. L. 2005, ApJ, 635, L73

Sandquist, E. L., \& Shetrone, M. D. 2003, AJ, 125, 2173

Sandquist, E. L., Bolte, M., \& Hernquist, L. 1997, ApJ, 477, 335

Sandquist, E. L., Latham, D. W., Shetrone, M. D., \& Milone, A. A. E. 2003, AJ, 125,810

Santolamazza, P., Marconi, M., Bono, G., et al. 2001, ApJ, 554, 1124

Sarajedini, A., \& Milone, A. A. E. 1995, AJ, 109, 269

Schönberner, D., \& Napiwotzki, R. 1994, A\&A, 282, 106

Schönberner, D., Andrievsky, S. M., \& Drilling, J. S. 2001, A\&A, 366, 490

Shara, M. M., Saffer, R. A., \& Livio, M. 1997, ApJ, 489, L59

Shara, M. M., Fall, S. M., Rich, R. M., \& Zurek, D. 1998, ApJ, 508, 570

Shetrone, M. D., \& Sandquist, E. L. 2000, AJ, 120, 1913

Siegel, M. H., Majewski, S. R., Cudworth, K. M., \& Takamiya, M. 2001, AJ, 121,935

Sills, A., \& Bailyn, C. D. 1999, ApJ, 513, 428

Sills, A., Bailyn, C. D., \& Demarque, P. 1995, ApJ, 455, L163

Sneden, C., Preston, G. W., \& Cowan, J. J. 2003, ApJ, 592, 504

Strom, K., \& Strom, S. 1970, ApJ, 162, 523

Stryker, L. L. 1993, PASP, 105, 1081

Templeton, M., Basu, S., \& Demarque, P. 2002, ApJ, 576, 963

van den Berg, M., Verbunt, F., \& Mathieu, R. D. 1999, A\&A, 347, 866

van den Berg, M., Orosz, J., Verbunt, F., \& Stassun, K. 2001, A\&A, 375, 375

van den Berg, M., Tagliaferri, G., Belloni, T., \& Verbunt, F. 2004, A\&A, 418, 509

von Braun, K., \& Mateo, M. 2002, AJ, 123, 279

Webb, N. A., Serre, D., Gendre, B., et al. 2004, A\&A, 424, 133

Wheeler, J. C. 1979, ComAp, 8, 133

Yan, L., \& Reid, I. N. 1996, MNRAS, 279, 751

Zoccali, M., Renzini, A., Ortolani, S., Bica, E., \& Barbuy, B. 2001, AJ, 121, 2638 